\title{
Journées annuelles de la Société française de gériatrie et gérontologie
}

\author{
G. Berrut (président de la SFGG) • O. Hanon (président du conseil scientifique de la SFGG) \\ C) Springer-Verlag France 2012
}

Chers Collègues et Amis,

Les Journées annuelles de la Société française de gériatrie et gérontologie se tiennent à Paris pour la $32^{\mathrm{e}}$ année consécutive. Cette réunion est devenue le rendez-vous incontournable de la gériatrie et de la gérontologie française. En cette année olympique, le congrès s'annonce lui aussi celui de tous les records. Le nombre d'abstracts proposés n'a jamais été aussi important témoignant de l'intérêt croissant des professionnels concernés et passionnés par le vieillissement dans ses dimensions gériatriques et gérontologiques.

Le programme scientifique a été établi afin de donner à cette réunion un caractère d'excellence dans les domaines de la recherche fondamentale, de l'épidémiologie, de la clinique, de la pharmacologie, des thérapeutiques mais aussi des sciences humaines et sociales, afin de favoriser les échanges entre chercheurs et cliniciens dans le domaine de la gériatrie et de la gérontologie.

Pour favoriser au maximum la diffusion et l'échange des informations, le programme comporte toutes les modalités qui ont fait la preuve de leur efficacité : sessions de formation médicale continue, mises au point, tables rondes, symposia de l'industrie pharmaceutique et bien sûr communica- tions orales et affichées sur les travaux originaux de recherche ou les expériences de terrain.

Tous les domaines cliniques de la gériatrie et de la gérontologie seront abordés pendant ces trois jours : pathologies cardiovasculaires, pathologies et risques infectieux, pathologies psychiatriques, pathologies digestives, pathologies neurologiques, l'oncogériatrie, les syndromes gériatriques, la fragilité, l'évaluation gériatrique, ainsi que les sciences humaines et sociales, l'organisation des soins, l'évaluation des pratiques, les droits de l'homme, la médiation. Nous ferons le point sur le déploiement national du programme MobiQual porté par notre société, avec le soutien de la CNSA.

Nous souhaitons que ces $32^{\text {es }}$ journées annuelles de notre société soient l'occasion, pour vous toutes et tous, de prendre une part très active à cette aventure de la gériatrie et de la gérontologie.

Nous vous y invitons chaleureusement en espérant vous voir aussi nombreux que pour l'édition 2011 qui a rassemblé plus de 1200 participants.

Rendez-vous à Paris, des 2 au 4 octobre 2012.

\footnotetext{
G. Berrut $(\bowtie)$

Pôle de gérontologie clinique, CHU Nantes, Nantes, France

e-mail : gilles.nantes@chu-nantes.fr

O. Hanon

Hôpital Broca, 54-56, rue Pascal, F-75013 Paris, France
}

Co-édition avec L'Année gérontologique (2012) 